\title{
Impacto de las relaciones humanas en la competitividad de las asociaciones
}

\section{Impact of human relations on the competitiveness of the associations}

\author{
Armando Pulido-Garzón ${ }^{1 a}$, Marlene Guerrero-Julio ${ }^{1 b}$, Yerlith Celis-Patiño ${ }^{1 \mathrm{c}}$ \\ ${ }^{1}$ Grupo de Investigación en Nuevas Tecnologías, Ingeniería Industrial, Universidad de Santander (Udes), Colombia. \\ Correos electrónicos: adir.industrial@udes.edu.co, ${ }^{\mathrm{b}}$ marlene.guerrero@udes.edu.co c $\mathrm{y} . c e l i s @$ unisimonbolivar.edu.co
}

Recibido: 18 diciembre, 2017. Aceptado: 12 marzo, 2018. Versión final: 20 junio, 2018.

\begin{abstract}
Resumen
El surgimiento de la teoría de las relaciones humanas en la administración incorporó aspectos influyentes en la productividad empresarial, como el clima organizacional y su impacto en el comportamiento y eficiencia de las personas que conforman la empresa. Elementos como los enmarcados en el factor $\mathrm{C}$, sumados a un modelo de negocios estructurado, propician una mejora continua en los procesos y en el sentimiento de bienestar y sentido de pertenencia de las personas. Este artículo presenta un modelo que, a través de técnicas de aprendizaje experiencial, permite el diagnóstico de los factores internos y externos que pueden afectar la productividad empresarial, desde el punto de vista de las relaciones humanas, identificando los paradigmas limitantes que impiden un avance y desarrollo sostenido.
\end{abstract}

Palabras clave: administración; capital humano; competitividad; cultura organizacional; modelo de negocio.

\begin{abstract}
The emergence of human relations theory in management incorporated influential aspects of business productivity such as the organizational climate and its impact on the behavior and the efficiency of the people who constitutes the company. Elements such as those framed in Factor C, combined with a structured business model, lead to a continuous improvement in the processes, in the sense of well-being and in the sense of belonging of the people. This article presents a model that through experiential learning techniques allows for the diagnosis of internal and external factors that can affect business productivity from the point of view of human relations by identifying the limiting paradigms that impede progress and sustainable development.
\end{abstract}

Keywords: administration; business model; competitiveness; human capital; organizational culture.

\section{Introducción}

En la actualidad las empresas se enfrentan a un nuevo paradigma económico, en el que su competitividad, además de depender de su participación en el mercado, la reducción de costos y el acceso a tecnología de escala, depende directamente de las personas y de su capacidad para generar valor agregado, a través de aspectos como la innovación, el incremento de la productividad y las relaciones con los clientes [1] [2].

De acuerdo con [3], [4] y [5], el comportamiento y la productividad de los miembros de la organización dependen de la percepción que ellos poseen sobre los factores organizacionales relacionados con el modelo de negocio de la empresa, la ejecución de sus actividades, las relaciones con otros miembros de la organización y

ISSN impreso: 1657 - 4583, ISSN en línea: 2145 - 8456, CC BY-ND 4.0 (c) () $९$

Como citar: A. Pulido-Garzón, M. Guerrero-Julio, Y. Celis-Patiño, "Impacto de las relaciones humanas en la competitividad de las asociaciones," Rev. UIS Ing., vol. 18, no. 1, pp. 61-72, 2019. doi: 10.18273/revuin.v18n1-2019005 
las experiencias resultantes de su quehacer diario. Estos factores comúnmente configuran el denominado clima organizacional, que genera un ciclo causal entre su percepción por parte de las personas y su comportamiento, es decir, el clima organizacional determina el sentido de pertenencia, comportamiento y productividad de las personas, pero estos, a su vez, según [6] y [7], determinan la calidad de los servicios prestados, la ejecución eficiente y efectiva de los procesos y, en general, el impacto y desempeño organizacional.

El clima laboral puede convertirse en fuente positiva de la productividad de las personas cuando genera confianza $\mathrm{y}$ fortalece su sentido de pertenencia hacia la organización y hacia sus objetivos estratégicos [4] [8]. En caso contrario, se pueden generar fluctuaciones laborales y factores de riesgo que inciden en el ausentismo, la ineficacia y el bajo rendimiento, asociado con la falta de correspondencia entre las metas personales y las metas del negocio.

El liderazgo juega entonces un papel fundamental en la mejora continua de los procesos y en el fortalecimiento del clima laboral, que influye en el sentimiento de bienestar y sentido de pertenencia de las personas [9] [10] [11]. Los gerentes de las organizaciones deben generar estrategias que permitan diagnosticar los diferentes elementos internos y externos que pueden afectar la productividad empresarial, desde el punto de vista de las relaciones humanas, identificando los paradigmas limitantes que impiden un avance y desarrollo sostenido [12].

Es en este marco en el que se ubica el modelo propuesto, que, al aplicar estrategias experienciales, posibilita que la organización tenga una percepción del relacionamiento interno y externo, con el fin de detectar las amenazas, las oportunidades, las debilidades y las fortalezas, que permitan identificar su posición competitiva para así priorizar las estrategias de mejora necesarias.

\section{Un acercamiento al modelo de diagnóstico competitivo}

El modelo planteado fundamenta sus resultados en una primera etapa en la que se realiza un diagnóstico del relacionamiento interno y un diagnóstico del relacionamiento externo (ver figura 1). En el ámbio

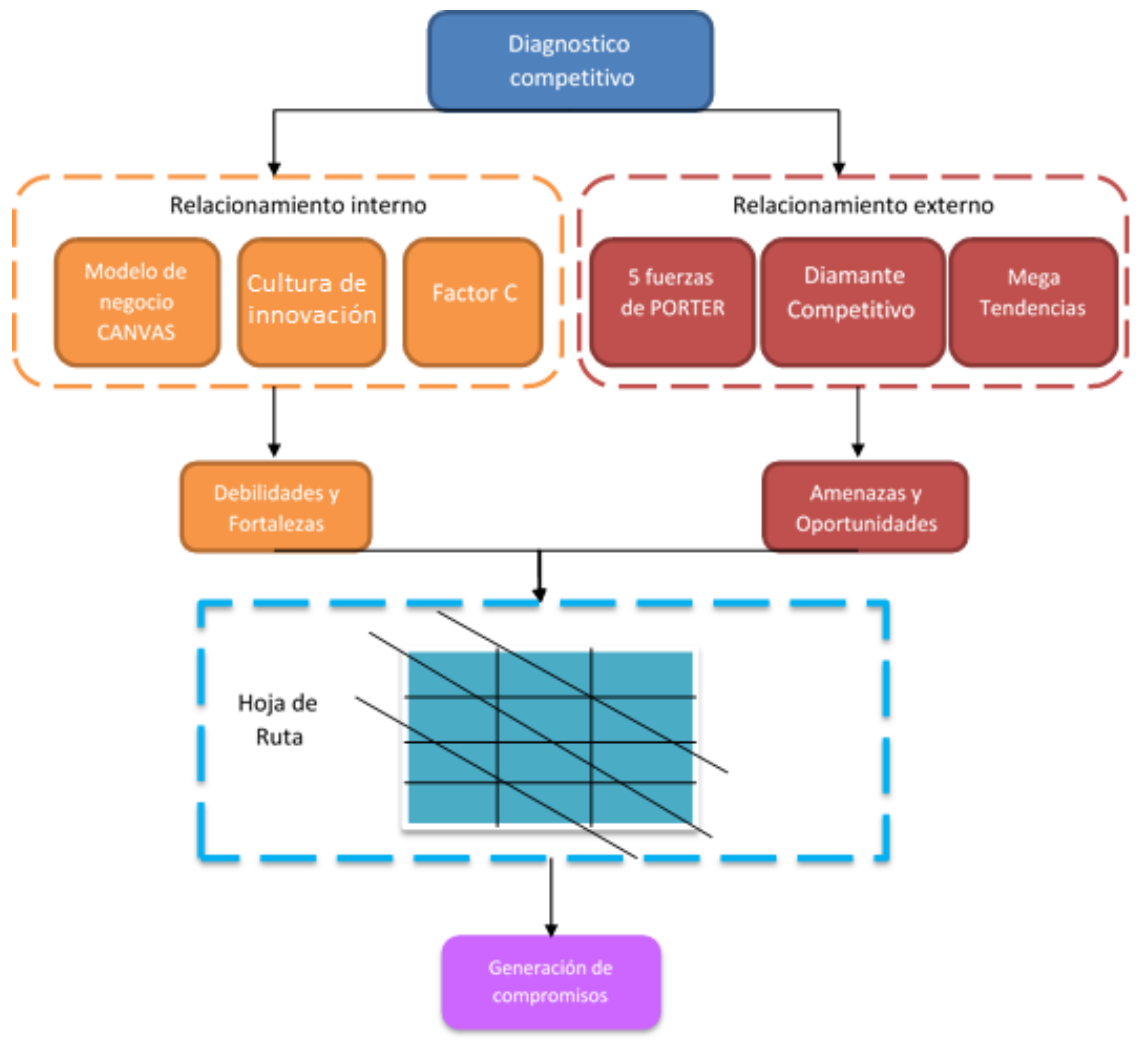

Figura 1. Modelo de diagnóstico competitivo. Fuente: elaboración propia. 
interno, el diagnóstico evalúa el modelo de negocio en torno a los elementos definidos en el modelo Canvas, el cual permite conocer la naturaleza del modelo de negocios [13]. En este modelo se evalúan aspectos tales como la propuesta de valor, el segmento de clientes, los canales de distribución, la relación con los clientes, el flujo de ingresos, los recursos claves, las actividades claves, la relación con aliados estratégicos y la estructura de costos (ver figura 2). Cada uno de estos elementos es evaluado en el ámbito interno de manera particular. Así, por ejemplo, para el caso de la propuesta de valor, se analizan aspectos como lo siguientes:

- La investigación e identificación continua de cambios en los servicios o productos ofrecidos.

- La propuesta de valor y su correspondencia con las necesidades o expectativas de los clientes.

- La coherencia de la definición de atributos claves de la empresa con lo que pide el cliente.

La manera indicada de leer las gráficas que resultan de la aplicación de cada uno de los instrumentos del modelo de diagnóstico es que, cuanto más se acerque el punto analizado al centro, más debilidad se tiene en el aspecto en mención. Por el contrario, si el punto se acerca a los extremos, la condición será de fortaleza. Se pude evidenciar, entonces, en el ejemplo presentado anteriormente, que una debilidad marcada en cuanto al modelo de negocio de la empresa analizada es la estructura de costos. La calificación se interpreta de la siguiente manera: de 0 a 2,99 es debilidad y de 3 a 6 es fortaleza, esto quiere decir que la debilidad tendrá más grado de criticidad en la medida en que más baja sea la calificación, y, por ende, la fortaleza estará más marcada, cuánto más alta sea la calificación.

De acuerdo con [14], «una empresa puede aumentar su capacidad de innovación si dedica suficientes recursos y capacidad directiva para gestionar el proceso de innovación». Es por ello que el segundo elemento en el ámbito interno, y abordado por el modelo propuesto, es la cultura de la innovación, que se enfoca en identificar los estilos de comportamiento de la organización y orienarlos hacia la innovación [15] [16].

Continuando con la lectura de la gráfica anterior, se puede analizar que una debilidad en la que debe trabajar la empresa es la experiencia en innovaciones, mientras que una fortaleza manifiesta es la participación en programas de formación.

El último elemento utilizado para diagnosticar el relacionamiento interno tiene que ver con el factor $\mathrm{C}$, bajo la percepción de que es el motor impulsor que permite aumentar la productividad en las asociaciones [17]. El factor $\mathrm{C}$ se soporta en ciertos valores que respetan y mantienen la esperanza en cada uno de los miembros de la organización, en pos del logro de un objetivo común y compartido. El instrumento diseñado permite identificar la solidez y la coherencia de este factor $\mathrm{C}$, pues evidencia todo aquello que en buena medida ha superado la organización, lo que aún les genera incomodidad y la visión conjunta de a dónde quieren llegar. De igual manera, a partir de este instrumento, se identifican los paradigmas limitantes que,

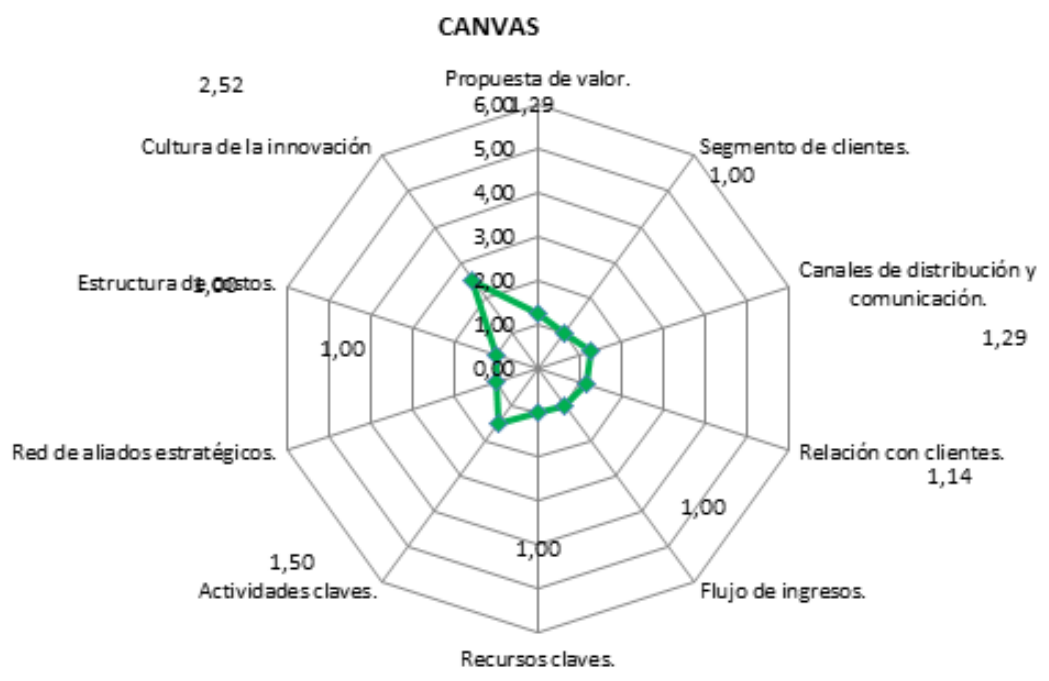

Figura 2. Ejemplo de diagnóstico Canvas. Fuente: elaboración propia. 


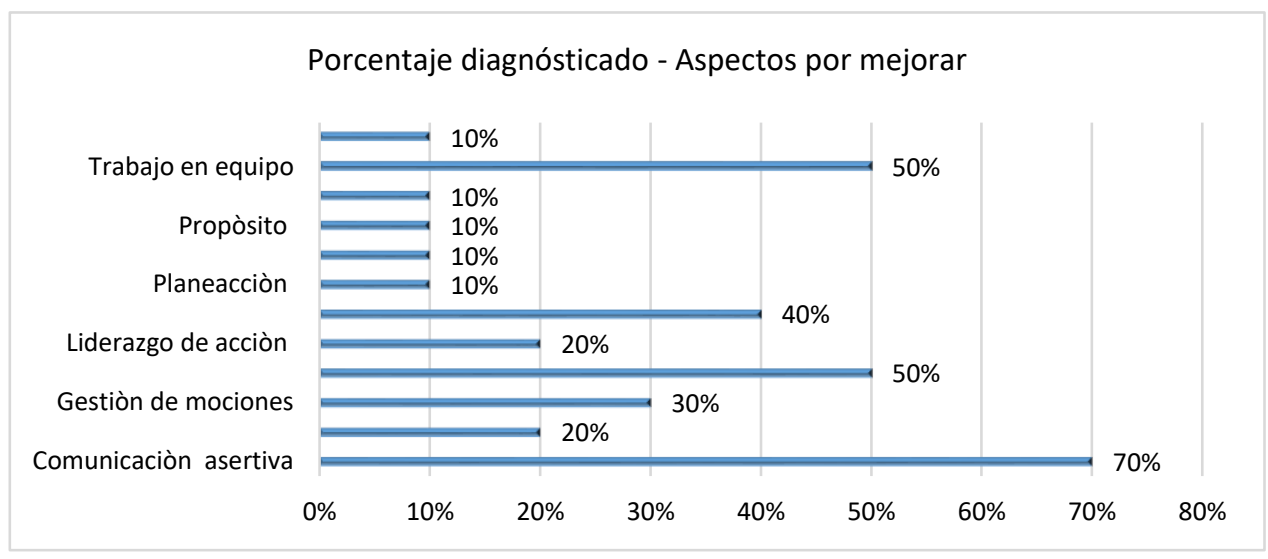

Figura 3. Modelo de diagnóstico competitivo. Fuente: elaboración propia.

con mayor fuerza, impiden un avance y un desarrollo sostenido (ver figura 3 ).

De forma paralela, el modelo propone realizar un diagnóstico del relacionamiento externo, en el cual se evaluarán las cinco fuerzas de Porter, a saber: entrada de nuevos competidores, poder de negociación de los clientes, poder de negociación de los proveedores, intensidad de la rivalidad y presión de productos/servicios sustitutos. La valoración de estas cinco fuerzas se enfoca en identificar la atractividad de un negocio en particular y establece qué tan competitivo es ese negocio. Para esto, las dos primeras fuerzas (barreras de entrada y productos sustitutos) definen el tamaño de margen estipulado como alto medio o bajo. Por su parte, el poder de negociación de cliente y los proveedores, sumados a la rivalidad, determinan quién en la cadena de valor se queda con el margen ganado. En resumen, el tamaño del margen y quién se queda con él reflejan la atractividad del negocio.

Así mismo, se evaluarán los elementos del diamante competitivo, que permite entender las ramificaciones sociales de la cadena de valor y comprender las dimensiones sociales del contexto competitivo de la empresa y los vínculos 'de afuera hacia adentro', que afectan su capacidad, y que son entendidos como las condiciones en las ubicaciones de una empresa (tales como la infraestructura de transporte y la política regulatoria honestamente aplicada) que afectan su capacidad para competir [18].

Finalmente, para terminar el diagnóstico externo, se analizan las megatendencias, que, según [19], están relacionadas con aquellos cambios que en los planos político, social, económico y cultural orientarán las

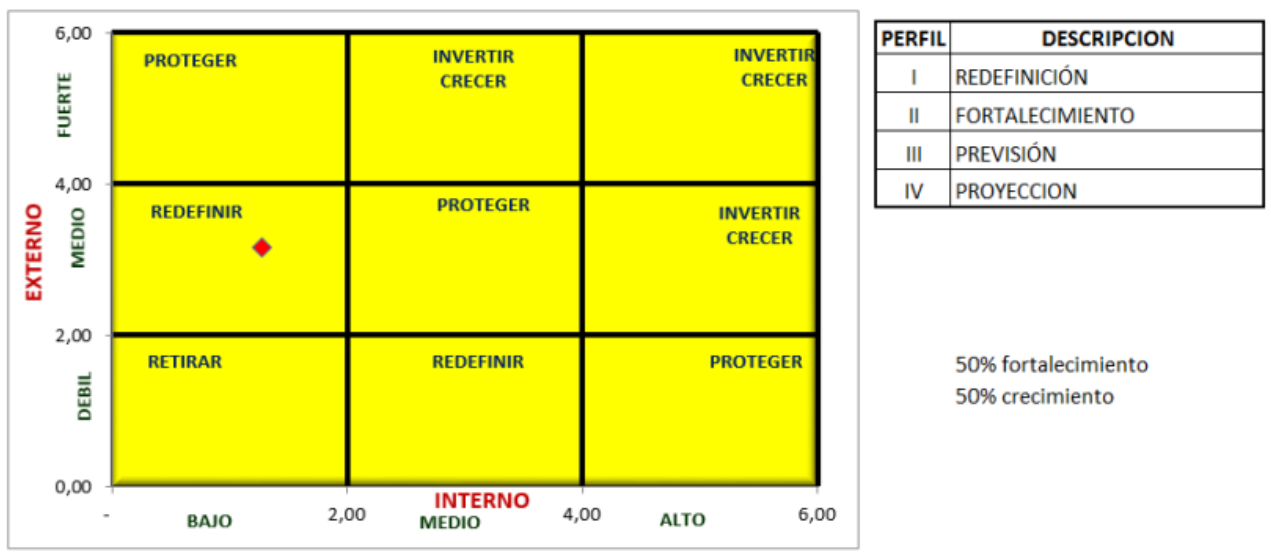

Figura 4. Posición competitiva. Fuente: elaboración propia. 


\section{Modelo de Negocios}

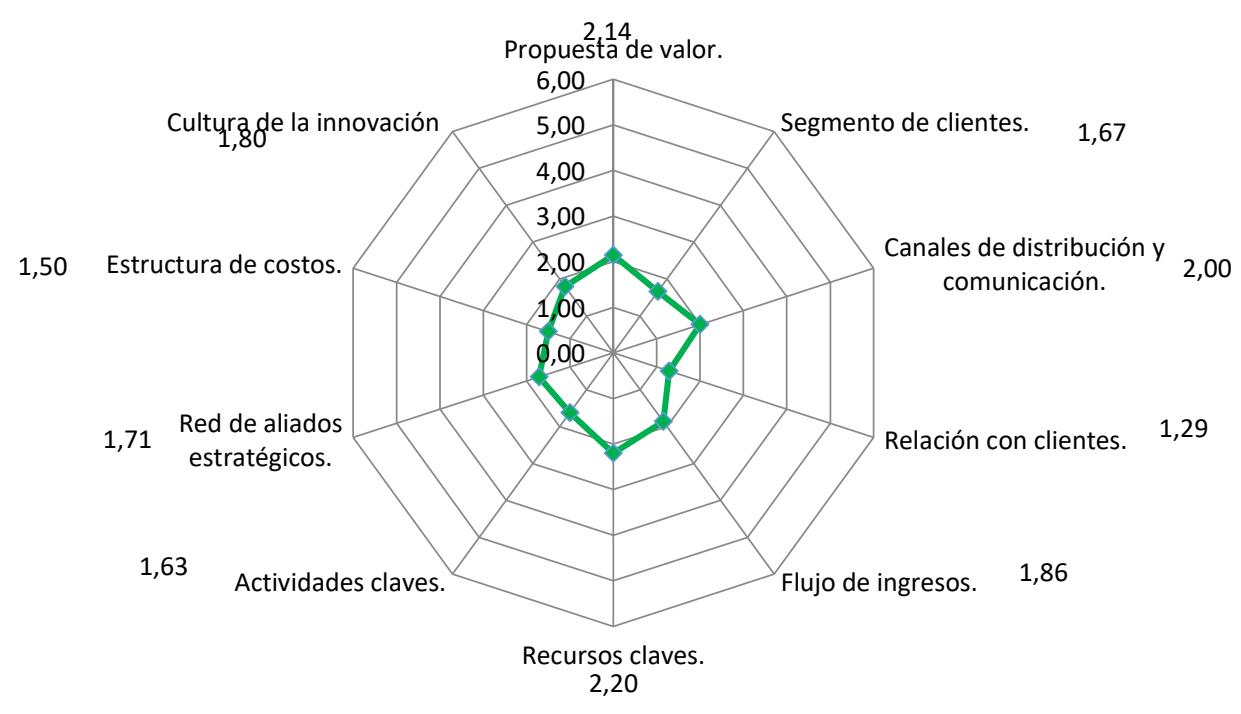

Figura 5. Diagnóstico del modelo de negocio de Asogatraganor.

tendencias en el ámbito mundial en un plazo de diez años o más, como lo hacen los big data, la innovación, la gestión de conocimiento, entre otras.

Para la interpretación del análisis externo, no se tiene una regla única que defina lo que está bien de lo que no, por esta razón es importante analizar cada contexto de manera independiente, con base en el análisis descrito en cada parámetro.

Como resultado del diagnóstico interno, se define un conjunto de debilidades y fortalezas, que, contrastadas con las amenazas y las oportunidades del diagnóstico externo, permitirán trazar una hoja de ruta en la que se identifique la posición competitiva de la organización, de modo que, con base en ello, se pueda generar una serie de estrategias que se deben convertir en compromisos organizacionales.

La posición competitiva permite establecer las siguientes posibles acciones en la organización:

- Retirar. Las organizaciones enmarcadas en este dominio requieren una completa reingeniería, y normalmente su baja atractividad implica un replanteamiento del modelo de negocios. En caso de tomar una decisión de continuar con el mismo modelo de negocio, se recomienda encaminar el $90 \%$ de las estrategias hacia el fortalecimiento interno.

- Redefinir. Los modelos de negocios ubicados en estos cuadrantes cuentan con una atractividad media baja, y sus estrategias deben estar formuladas en una relación de $70 \%$ fortalecimiento y $30 \%$ crecimiento. Esto implica, en cuanto a fortalecimiento, crear estrategias de mejora interna de la empresa; en cambio, el crecimiento está relacionado con el aprovechamiento de sus capacidades para abordar las oportunidades del entorno.

- Proteger. Los modelos de negocios ubicados en estos cuadrantes cuentan con una atractividad media, y sus estrategias deben estar formuladas en una relación de $50 \%$ fortalecimiento y $50 \%$ crecimiento.

- Invertir/crecer. Cuando sus valores internos y externos son lo suficientemente fuertes como para competir en el mercado con una propuesta de valor diferenciada. Normalmente una organización ubicada en esta zona debe enfocarse más en estrategias de crecimiento, antes que en el fortalecimiento interno.

Los porcentajes estimados para la formulación de estrategias pueden variar, según la interpretación del analista, y son una referencia para el logro de un equilibrio competitivo de la organización. 


\section{Una perspectiva de la puesta en marcha del modelo en una asociación de Norte de Santander}

Como se ha analizado, el clima organizacional es determinante de la productividad y la competitividad. De acuerdo con [17], esto cobra mayor relevancia para las asociaciones, puesto que la mayoría de ellas involucra el denominado factor $\mathrm{C}$, que suma conceptos como cooperación, compañerismo, comunidad, compartir, colectividad, carisma y comunión, como elementos de integración humana y crecimiento organizacional. A continuación, se presentarán los resultados de la aplicación del modelo en la asociación ganadera Asogatraganor, ubicada en el municipio de Gramalote en Norte de Santander Colombia. Esta asociación fue escogida en el marco de una estrategia de intervención de la agencia de cooperación alemana GIZ, que buscaba el mejoramiento del entorno regional de Norte de Santander, desde el fortalecimiento del trabajo asociativo.

De acuerdo con el modelo, el primer aspecto a evaluar es el relacionamiento interno, con base en el modelo de negocio, cultura de innovación y factor $\mathrm{C}$. Es importante resaltar que la metodología utilizada para el relevamiento de información en la asociación se desarrolló a través de técnicas de aprendizaje experiencial, en las que los asociados podían poner en evidencia los diferentes aspectos que se deseaban analizar. De acuerdo con [20], las técnicas de aprendizaje experiencial permiten conectar la realidad a través de metáforas de aprendizaje significativo. Estas tecnicas, basadas en el constructivimo, interconectan experiencia, conocimiento y comportamiento, y se adptan a diferentes estilos de aprendizaje. En otras palabras, permiten «aprender haciendo» [21]. Dentro de las técnica utilizadas con Asogatraganor se trabajaron esquemas de divergencia y convergencia, puesta en escena de aspectos positivos y por mejorar, lúdicas de liderazgo y confianza y proyección de lo que cada uno quiere ser. Con base en los resultados obtenidos, en Asogatraganor se pueden determinar varios aspectos por mejorar, a saber:

- Sobre el modelo de negocios. En términos generales, esta categoría carece de diferenciación, dado que la propuesta de valor está enfocada en proveer productos o insumos para ganadería como un commodity (producto sin valor agregado). Un negocio de estas características subsiste principalmente porque Asotraganor es la única asociación dedicada a este negocio en la zona, lo que genera ventajas competitivas que no son sostenibles, y, por ende, esto implica que no es un buen modelo de negocio. En

\section{Modelo de Negocios}

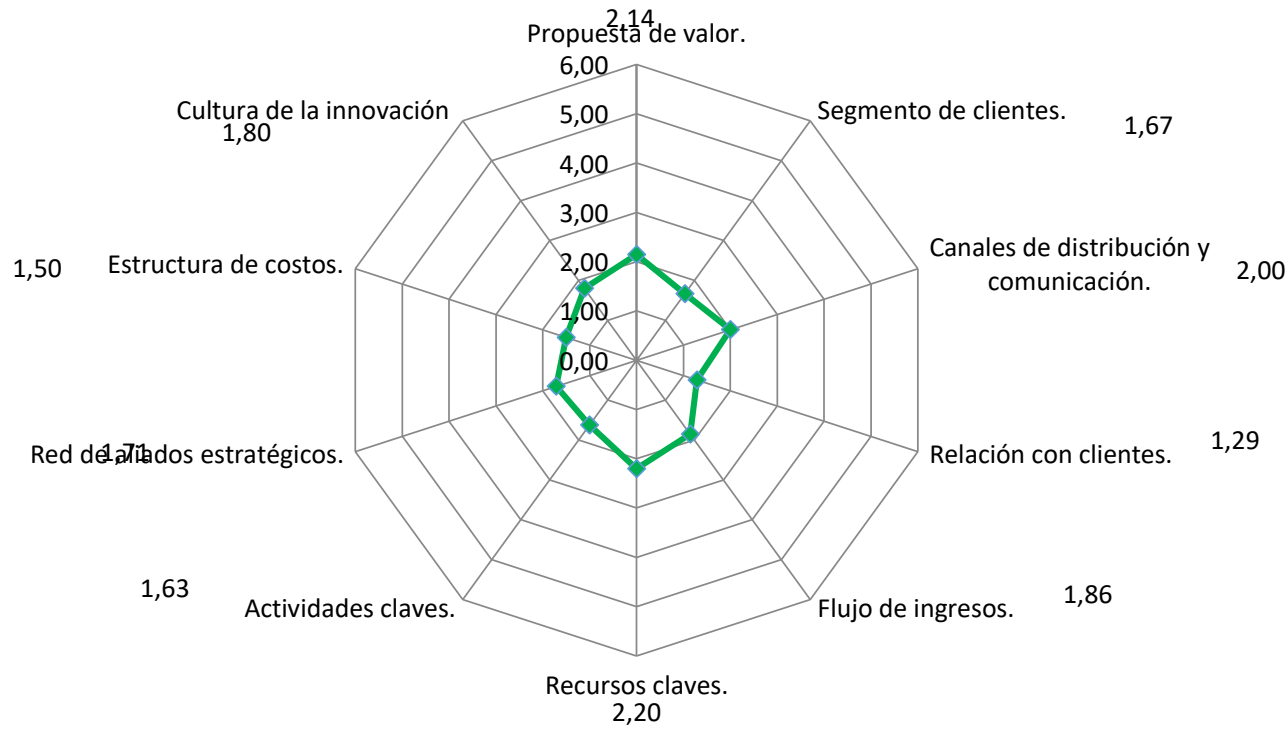

Figura 6. Resultados del diagnóstico a nivel de cultura de innovación. 
resumen, puede afirmarse que este contexto supone una debilidad media alta (ver figura 5).

- La propuesta de valor. Esta propuesta se basa en la comercialización de productos básicos que pueden ser reemplazados en cualquier momento con la llegada de un competidor que ofrezca los mismos productos o servicios. Sin embargo, la asociación presenta ciertas fortalezas con sus productos que pueden ser trasladados a nuevos y mejores segmentos de mercado. Por su parte, las debilidades también aparecen en este contexto, dado que la falta misma de documentación los está llevando a desconocer el negocio y les quita la posibilidad de sofisticar el servicio. En consecuencia, terminan entregando diferenciación.

- Segmento de clientes. Se evidencia una debilidad en el conocimiento del cliente actual, lo que, de acuerdo con [13 ], impide visualizar otro segmento de clientes, y afecta de manera directa la forma de percibir ingresos. Adicionalmente, la relación con los clientes plantea una debilidad alta, precisamente porque se comportan como un monopolio, que, por tener un mercado asegurado no se mueve a generar aspectos que enamoren al cliente actual y atraigan a otros nuevos. Asimismo, no cuentan con procesos de recolección de datos o mantenimiento de clientes, que les permitan identificar sus necesidades. En consecuencia, no se mueven hacia el mejoramiento de este criterio.

- Canales de comunicación con el cliente. En la actualidad, estos canales son suficientes para la propuesta de valor y el tipo de clientes. Sin embargo, esto no quiere decir que estén bien. Es necesario pensar en la sofisticación de la propuesta de valor, y esto traerá consigo clientes que implican nuevos canales de comunicación. Visto desde este punto de vista, la debilidad de este contexto es alta.

- Flujo de ingresos. El análisis de los elementos Canvas hasta aquí analizados permite comprender por qué los niveles de ingreso de la asociación no son los esperados, y si a esto se le suma una alta dependencia de las cuotas de asociación, se puede concluir que el modelo no es sostenible, y que impide el crecimiento de la asociación.

- Recursos claves. En este aspecto es importante resaltar que el talento humano tiene fortalezas medias, y, en términos de cantidad, el recurso humano no es suficiente, dado que está constituido principalmente por un par de personas. Su distancia con la tecnología hace que tengan grandes debilidades que les impedirá crecer a futuro.

- Actividades claves. Aunque ticenen claro cuáles son las actividades que llevan a cabo para generar su producto, estas no son las que deberían tener para aprovechar nuevos y mejores modelos de negocio. En este sentido, es importante hacer una reingeniería de actividades que permitan generar valor. A partir de lo anterior, este aspecto presenta una debilidad media alta.

- Relación con aliados estratégicos. No cuentan con procesos formales de exploración y generación de alianzas. En este sentido, se debe destacar que es importante fortalecer su proceso colaborativo entre asociados, para luego abrirse a la consecusión de aliados que les permita crecer. Esto constituye una debilidad media alta.

- Estructura de costos. Los costos son, sin duda alguna, una debilidad alta, en primer lugar, porque no tienen conciencia de su existencia e impacto, y, en segundo lugar, porque superan los ingresos y generan un modelo de negocios insostenible en el tiempo. La debilidad es alta.

El segundo aspecto a evaluar, en el marco del relacionamiento interno en el modelo, es la cultura de la innovación. En este contexto, hay muchos aspectos por mejorar, lo que indica que es una debilidad alta. Sin embargo, se le puede dar un cambio significativo, principalmente, porque, de acuerdo con [22], tienen una tendencia a la participación en procesos de formación que podría aprovecharse (ver figura 6).

En lo concerniente al diagnóstico con el factor $\mathrm{C}$, se puede decir que se encuentran en un nivel de aceptación importante por parte de la asociación, y que ella misma ha seleccionado los siguientes determinantes:

- Dinamismo. Como equipo se mueven rápidamente hacia lo que requieran hacer para salir adelante. Están atentos a lo que se necesite para accionar.

- Liderazgo. Reconocen la necesidad de un liderazgo como elemento fundamental en el quehacer diario. Se han organizado de tal manera que existan estos líderes de manera visible.

- Pensamiento positivo. Se logra identificar la resiciliencia como un aspecto diferenciador en la asociación, dado que se detecta la capacidad de comprender los diferentes momentos de crisis como oportunidades.

- Unión. Tienen claro que para llegar a la meta se necesitan unos a otros, y, por tanto, se organizan y buscan estrategias para que de una u otra forma todos estén juntos.

La organización tiene claro que necesita apoyo de entidades grandes y que es necesario realizar alianzas que le permitan crecer, dado que es una asociación dinámica que siempre está buscando apoyo para su posicionamiento. Esta asociación busca el contacto con personas y con organizaciones que les permitan llegar al 
éxito, además maneja conceptos básicos de liderazgo, como estrategia de unión y avance en equipo.

Los elementos del factor $\mathrm{C}$ generan oportunidades de mejora en un tiempo relativamente rápido, lo que permite mantener la esperanza en el éxito. Dentro de esos elementos se encuentran los siguientes:

- Chisme: envidia y mentira: estos vicios se presentan de manera recurrente, y generan un desgaste emocional, ya que no poseen herramientas para manejarlos y, en consecuencia, se genera desmotivación.

- Respeto: existe una marcada tendencia a validar únicamente una sola forma de realizar las cosas, invalidando fácilmente los ritmos, las opiniones y las acciones de la mayoría, privilengiando los del líder y dejando de lado mecanismos como la generación de acuerdos.

- Trabajo en equipo: la asociación presenta motivación y una tendencia a esforzarse por el logro de los objetivos comunes, sin embargo, el sistema de compensación natural de la asociación tiende a generar sensaciones de insatisfacción, al no ser equitativos los reconocimientos.

Los miembros de la asociación reconocen a Asogatraganor, tienen la convicción de que será una asociación con posicionamiento económico y que cada miembro alcanzará sus sueños, por querer aplicar el esquema de ganar - ganar [23] con sus asociados, clientes $\mathrm{y}$ todas las personas y organizaciones con quienes se relacionen. Por tanto, para apoyar esta visión de la asociación, se hace necesario realizar procesos de aprendizaje experiencial, relacionados con la confianza, como pilar del trabajo en equipo; la comunicación asertiva, para que todos los miembros de la asociación se articulen a partir de un bien común, $y$, por ultimo, el liderazgo, para asegurar el aprendizaje de todos los asociados, y que, de esa manera, puedan aparecer y accionar líderes situacionales, en el momento que lo requieran. Esto representa una ventaja competitiva y una disminución en el nivel de esfuerzo de la asociación.

Por su parte, en cuanto a los resultados del análisis externo, se puede evidenciar que este es un negocio con una atractividad media baja. Esto se debe la presencia de bajas barreras de entrada y muchos productos sustitutos, que logran bajos márgenes, los cuales son principalmente para el cliente y otra buena parte para la asociación, precisamente por el carácter de monopolio que presentan, que no es sostenible.
El modelo de negocio actual es fácilmente reemplazable, porque se enfoca en la comercialización de productos para el ganado bovino. Esto abre la puerta a la llegada de nuevos competidores que, con barreras de entrada más altas o diferenciación, podrían sustituir su propuesta de valor y dejar el negocio fuera de competencia.

Por su parte, los clientes tienen una posición intermedia de negociación, debido a que una parte significativa de ellos son los mismos asociados, indicando que es imperativo generar la búsqueda de nuevos clientes que impliquen otras formas de hacer negocios. En este sentido, se puede afirmar que existen más oportunidades que amenazas, pero esto cambiará en la medida en que ingresen a nuevos segmentos de mercado.

$\mathrm{La}$ asociación tiene oportunidades medias-altas, principalmente porque está constituida como un monopolio, pero como la idea es llegar a nuevos segmentos, esto traería consigo nuevos retos en términos de rivalidad; por ahora, es momento de fortalecerse y prepararse para el cambio.

En cuanto a la entrada de nuevos competidores, esto es una amenaza alta, principalmente porque en el mercado global existe una diversidad muy amplia de productos sustitutos como los productos y los procesos orgánicos. Esto imprime más presión a la necesidad de sofisticar la propuesta de valor.

Ahora bien, existen oportunidades de formación, pero todas ellas están en la cabecera principal del departamento de Norte de Santander, lo cual genera a su vez una amenaza. Por su parte, el estado de las vías y el acceso a la tecnología son en sí una amenaza que le hace perder competitividad. En este sentido se puede afirmar que tiene una amenaza media alta.

Los resultados generados en los diagnósticos permiten representar la hoja de ruta de Asogatraganor, la cual obtuvo una puntuación de 1,78 de 6 en el contexto interno y 3,69 de $6 \mathrm{n}$ el contexto externo. La posición competitiva indica que la asociación está en un punto de redefinición, esto quiere decir que debe fortalecerse desde adentro inicialmente antes que salir a competir (ver figura 7).

Con base en la hoja de ruta, se generan un conjunto de compromisos a nivel de estrategias que la asociación debe ejecutar, con el fin de lograr la redefinición planteada. 
Tabla 1. Estrategias de mejora para la redefinición de Asogatraganor.

\begin{tabular}{|c|c|}
\hline Estrategia & Descripción \\
\hline $\begin{array}{l}\text { Desarrollar un programa de } \\
\text { formación dedicado a enseñarle al } \\
\text { productor la medición del proceso, } \\
\text { para que pueda llevar una } \\
\text { trazabilidad y una caracterización } \\
\text { (zonas, alturas, tipo de tierra, raza, } \\
\text { insumos empleados etc.). }\end{array}$ & $\begin{array}{l}\text { Este programa implica que el productor comprenda la importancia de conocer toda la } \\
\text { información pertinente de su modelo de negocio, sobre cómo documentar la } \\
\text { información, que será la base que le permita la mejora continua, y cómo aplicar la } \\
\text { metodología en el logro de los proyectos que están consignados en la presente } \\
\text { estrategia. }\end{array}$ \\
\hline $\begin{array}{l}\text { Desarrollo de programa de buenas } \\
\text { prácticas de ordeño, focalizado en } \\
\text { producción orgánica, buen manejo } \\
\text { ambiental, buenas prácticas } \\
\text { sociales y trazabilidad de } \\
\text { producto. }\end{array}$ & $\begin{array}{l}\text { En la actualidad gran parte de los asociados no tienen en sus fincas la implementación } \\
\text { de las buenas prácticas de ordeño. En consecuencia, se plantea este programa para } \\
\text { orientar y desarrollar capacidades en los asociados sobre cómo obtener una buena } \\
\text { leche cruda. Para esto es importante que el asociado comprenda y aplique en el ganado } \\
\text { las siguientes variantes: alimentación de forma adecuada, bienestar y equilibrio con } \\
\text { el medioambiente, para tener como resultado una leche sana. De la misma forma, el } \\
\text { campo se caracteriza por no presentar formas adecuadas en los procesos de } \\
\text { contratación del talento humano, por ende, es importante incluir un programa de } \\
\text { buenas prácticas sociales que garanticen el bienestar de las personas. }\end{array}$ \\
\hline $\begin{array}{l}\text { Desarrollo de programa de } \\
\text { formación, para el desarrollo de } \\
\text { productos de alto valor, como } \\
\text { quesos añejos, productos } \\
\text { "cuchareables" tipo gourmet, etc. }\end{array}$ & $\begin{array}{l}\text { A la fecha, las asociaciones se dedican a ser proveedores de la empresa Leche la } \\
\text { Mejor; mediante la producción de leche cruda, lo cual no les genera muy buenas } \\
\text { ganancias, por ende, el objetivo del proyecto es desarrollar la creatividad y la } \\
\text { innovación de los productores en la investigación e implementación de nuevos } \\
\text { productos, con el fin de que a mediano plazo los asociados dejen de ser proveedores } \\
\text { de Leche la Mejor, y empiecen a ofrecer un productos diferenciadores, que generaran } \\
\text { mejores ganancias, como quesos añejos y productos "cuchareables". }\end{array}$ \\
\hline $\begin{array}{l}\text { Desarrollo de programa de } \\
\text { marketing especializado, } \\
\text { orientado a identificar el mercado } \\
\text { más apropiado, según el tipo de } \\
\text { producto. Esto debe incluir } \\
\text { desarrollo de redes sociales, sitio } \\
\text { web, construcción de historias, } \\
\text { entre otros. }\end{array}$ & $\begin{array}{l}\text { Los productores desconocen por completo lo que pasa con el cliente final, esto se } \\
\text { debe en gran medida a que no tienen contacto con él. En consecuencia, los productores } \\
\text { no se orienten al logro de la satisfacción del cliente y pierdan la posibilidad de conocer } \\
\text { y desarrollar mejoras en la propuesta de valor. } \\
\text { El siguiente proyecto busca precisamente que los productores cierren esa brecha de } \\
\text { conocimiento, mediante la incorporación de acciones orientas al marketing } \\
\text { especializado. Esto significa que el productor desarrollará el producto en óptimas } \\
\text { condiciones, guiado por la inteligencia de mercadeo, o sea, que tendrá la metodología } \\
\text { para comprender qué pasa con el cliente y cuáles son sus necesidades. }\end{array}$ \\
\hline $\begin{array}{l}\text { Especializar las fincas mediante el } \\
\text { desarrollo de programas de } \\
\text { caracterización de tierras y razas, } \\
\text { que permitan identificar cuál es el } \\
\text { producto más indicado en relación } \\
\text { costo-beneficio para desarrollarlo. }\end{array}$ & $\begin{array}{l}\text { En la actualidad, las fincas o las tierras presentan diferentes alturas, condiciones } \\
\text { climáticas, caracterización de suelos, entre otros aspectos. De esta forma, los } \\
\text { productores se enfocan en tener las mismas reses, sin importar las diferencias } \\
\text { mencionadas, y, por ende, la productividad varía en todos los casos. Esto mismo abre } \\
\text { la necesidad de caracterizar las reses, con el fin de identificar aquellas reses que } \\
\text { permitíían lograr mejores resultados y, de esta forma, obtener un nivel de } \\
\text { especialización que haga más productiva a la empresa. }\end{array}$ \\
\hline $\begin{array}{l}\text { Desarrollo de marca de producto } \\
\text { Gramalote o Norte de Santander. } \\
\text { Esta marca debe incluirse dentro } \\
\text { del branding del producto. }\end{array}$ & $\begin{array}{l}\text { El desarrollo de la marca del producto permite identificar los productos de la región } \\
\text { bajo una sola marca que facilite su venta y promoción en diferentes mercados. El } \\
\text { desarrollo de esta marca es el principio de la recuperación del orgullo regional y la } \\
\text { incorporación de sentido de pertenencia hacia lo nuestro, por ende, es importante } \\
\text { contar con la participación de todos los asociados, entes de gobierno, entidades de } \\
\text { apoyo, entre otras, en la definición de los aspectos a incorporar en la marca. Este } \\
\text { proceso estará acompañado por expertos en diseño e incorporación de marcas. }\end{array}$ \\
\hline
\end{tabular}




\section{Conclusiones}

El diagnóstico competitivo planteado en el modelo propuesto, permite la identificación de aquellos aspectos del relacionamiento interno y externo que pueden afectar de manera positiva o negativa el posicionamiento competitivo de una organización. Su aplicación en la asociación Asogatraganor, ayudó a establecer los elementos que a nivel de modelo de negocio, cultura de la innovación y factor $\mathrm{C}$, eran determinantes de las debilidades y oportunidades para su fortalecimiento. Así mismo, se pudieron evidenciar a nivel externo los aspectos de actractividad, relacionamiento con clientes y proveedores, análisis de la competencia y oportunidades de formación, que pueden generar amenazas o fortalezas que afecten su crecimiento.

El modelo planteado en este artículo es el resultado de un proceso de revisión consciente de las características internas y externas que pueden afectar la posición competitiva de una organización y por ello puede ser aplicable a diferentes sectores, obteniendo resultados específicos.
El impacto de las relaciones humanas en la competitividad de las asociaciones es cada vez más representativo, dado que estas afectan no solo su fortalecimiento, sino también su crecimiento. Aspectos como el conocimiento de lo que necesita el cliente y el establecimiento de canales adecuados de comunicación con este, la potencialización de las competencias del recurso humano, el liderazgo, el pensamiento positivo, el trabajo en equipo, entre otros, juegan un papel fundamental a la hora de comprender si es necesario retirar un negocio del mercado, redefinirlo, protegerlo o invertir en su crecimiento.

\section{Agradecimientos}

Los autores del artículo agradecen a la asociación Asogatraganor, de Norte de Santander, en donde se llevó a cabo parte del proyecto que dio origen a esta publicación.

Así mismo, agradecen a la agencia de cooperación Alemana GIZ, por propiciar el desarrollo de proyectos que coadyuvan al mejoramiento del entorno regional de Norte de Santander, desde el fortalecimiento del trabajo asociativo.

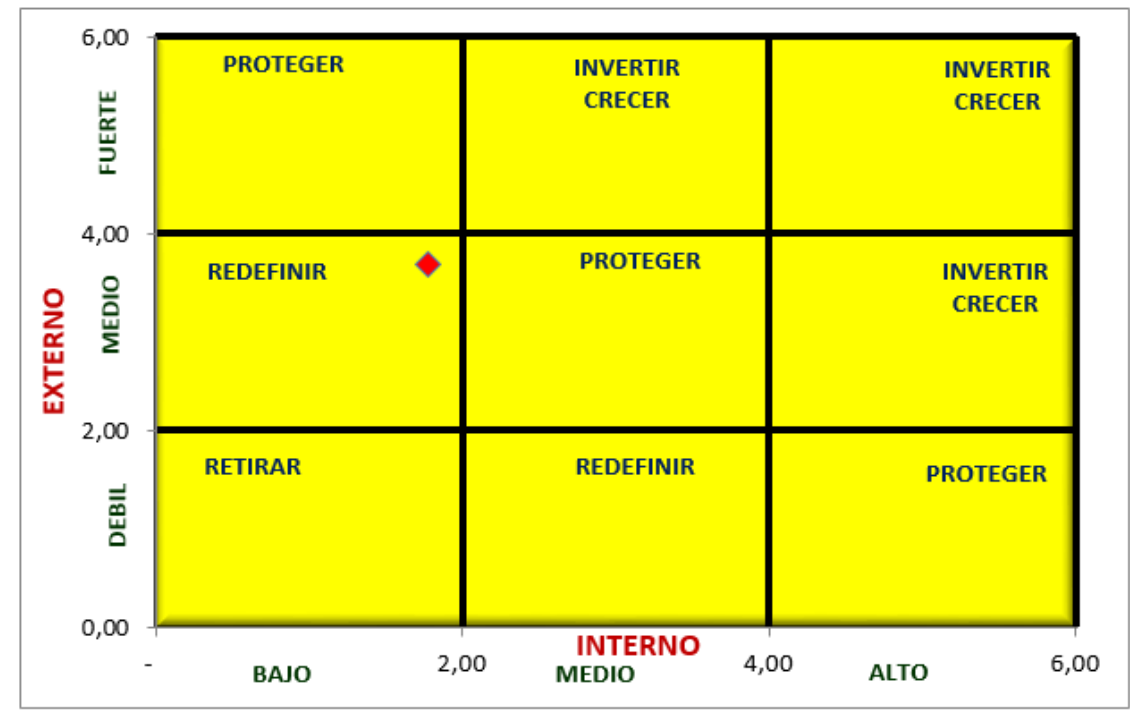

Figura 7. Posición competitiva de Asogatraganor.

\section{Referencias}

[1] G. Calderón, "Dirección de recursos humanos y competitividad," Revista Innovar, vol. 22, no. 22, pp. 157-172, 2003. doi: 10.1097/HMR.0b013e31825e718a
[2] T. Kijek and A. Kijek, "Is innovation the key to solving the productivity paradox?," Journal of Innovation \& Knowledge, 2018. doi: 10.1016/j.jik.2017.12.010 
[3] J. Salazar, J. Guerrero; Y. Machado y R. Canedo, "Clima y cultura organizacional: dos componentes esenciales en la productividad laboral," Revista ACIMED, vol. 20, no. 4, pp. 67-75, 2009.

[4] R. Shanker, R. Bhanugopan, B. Van der Heijdenbd and M. Farrell, "Organizational climate for innovation and organizational performance: The mediating effect of innovative work behavior," Journal of Vocational Behavior, vol. 100, pp. 67-77, 2017. doi: 10.1016/j.jvb.2017.02.004

[5] E. Venutolo, "Estudio del clima laboral y la productividad en empresas pequeñas y medianas: el transporte vertical en la ciudad autónoma de buenos aires", tesis doctoral, Universidad Politécnica de Valencia, España, 2009.

[6] M. Chiang, M. Martín y A. Núñez, Relaciones entre el clima organizacional y la satisfacción laboral. Madrid, España: Editorial Comillas, 2010.

[7] J. Ramírez, “Cultura organizacional e identidad en la nueva competitividad", Revista Innovar Journal Revista de Ciencias Administrativas y Sociales, vol. 12, no. 12, pp. 45-63, 1998.

[8] I. Chiavenato, Introducción a la teoría general de la administración. McGraw-Hill, 2006.

[9] H. Leroy, J. Segers, D. Dierendonck and D. Hartog, "Managing people in organizations: Integrating the study of HRM and leadership". Human Resource Management Review, vol. 28, no. 3, pp. 249-257, 2018. doi: 10.1016/j.hrmr.2018.02.002

[10]R. Vázquez, Habilidades directivas y técnicas de liderazgo: Su aplicación en la gestión de equipos de trabajo. España: Ideaspropias Editorial S.L., 2005.

[11] D. J. Hughes, A. Lee, A. W. Tian, A. Newman, y A. Legood, "Leadership, creativity, and innovation: A critical review and practical recommendations," Leadersh. Q., vol. 29, no. 5, pp. 549-569, 2018. doi: 10.1016/j.leaqua.2018.03.001

[12] M. Carcamo, Las relaciones humanas y la administración del personal. Santiago de Chile: Editorial Andres Bello, 1968.

[13] A. Osterwolder y Y. Pigneur, "Generación de modelos de negocios. Un manual para visionarios, revolucionarios y retadores. Bogotá : Planeta Colombia, 2017.
[14] Guía para gestionar la innovación, Reflexiones. Consejería de Ciencia, Tecnología, Industria y Comercio, San Cristóbal, 2016, pp. 14.

[15] P. Morcillo, Cultura e innovación empresarial: la conexión perfecta. México: Ediciones Paraninfo. S.A., 2007

[16] T. Michaelis, R. Aladin and J.Pollack, "Innovation culture and the performance of new product launches: A global study," J. Bus. Ventur. Insights, vol. 9, pp. 116127, 2018, doi: 10.1016/j.jbvi.2018.04.001

[17] M. Agudelo, B. Patiño y M. Ramos, "Conceptualización del factor C. Antecedentes y perspectivas", Revista Ingeniería Solidaría, vol. 6, no. 10, pp. 95-101, 2010.

[18] M. Kramer y M. Porter, "Estrategia y sociedad", Harvard Business Review, vol. 84, no. 12, pp. 45-56, 2006.

[19] F. López, América Latina 2020: escenarios, alternativas, estrategias. México: Temas Grupo Editorial, 2009.

[20] W. Haque, "Experiential Learning: Beyond the Classroom and Connecting with the Industry", en Tomorrow's Learning: Involving Everyone. Learning with and about Technologies and Computing, Springer, Berlin, Heidelberg, 2017, pp. 443-452.

[21] M. Romero-Ariza, "El aprendizaje experiencial y las nuevas demandas formativas", Revista de antropología experimental, vol. 10, pp. 89-102, 2010.

[22] A. Del Agulla y A. Padilla, "Factores derminantes de la innovación en empresas de economía social. La importancia de la formación y de la actitud estratégica". CIRIEC-España, Revista de Economía Pública, Social y Cooperativa, vol. 67, pp. 129-155, 2010.

[23] C. Mendieta y O. Vela, Ni tú ni yo: Cómo llegar a acuerdos. Barcelona, España: Editorial Grao, 2005. 